\title{
Application of Two-Level Joint Information Fusion Model in Intelligent Vehicle
}

\author{
http://dx.doi.org/10.3991/ijoe.v12i03.5154 \\ Yanting Lan ${ }^{1}$, Jinying Huang ${ }^{2}$ and Xiaodong Chen ${ }^{3}$ \\ ${ }^{1,2}$ North University of China,Taiyuan, China \\ ${ }^{3}$ China Agricultural University, Beijing ,China
}

\begin{abstract}
This paper proposes a two-level joint information fusion model combining BP neural network and D-S evidence theory. The model of great practical value reduces target identification error probability by multiple features of the target information, shows good scalability with its two steps of information fusion model, and conveniently increases/reduces feature fusion information source according to different situations and different objects. The method used for intelligent vehicles has good flexibility and robustness in tracking and avoiding obstacle. The simulation tests have verified effectiveness of the method.
\end{abstract}

Index Terms-information fusion, intelligent vehicle, neural network, D-S evidence theory

\section{INTRODUCTION}

A multi-feature fusion target recognition method based on neural network[1-3] and D-S evidence theory[4-6] is used in two levels in view of the uncertainty of sensor information in intelligent vehicle navigation. In the first level, the corresponding neural network carries on the character recognition of each kind of feature information among the variety of information extracted from the regional environment. In the second level, fusion decision of neural network recognition results is done with D-S evidence theory. The method of great practical value uses multiple features of the target information to reduce the probability of false target recognition, and uses two steps of information fusion model showing good scalability, and changes feature fusion information source according to different situations and different objects conveniently.

\section{TEST MODEL}

Specific configuration of the sensor is shown in Fig.1. The sensor of the vehicle consists of 2 CCD cameras, one gyroscope and 15 ultrasonic sensors. The CCD cameras are arranged in the front center of the vehicle, used to detect the deviation information and navigational line; the gyroscope is installed behind the two CCD cameras; the fourteen ultrasonic sensors are fitted in different positions, where 9 of them are mounted in the front according to the certain angle interval lined annular array, two in the rear, the other 6 in the left and right sides, respectively 2 in either side.

\section{BP NEUTRAL NETWORK}

Neural network fuses the information from ultrasonic sensors, where the center line of the sensors on two sides and the center line of center sensor form an angle of $30^{\circ}$ with each ultrasonic sensor placed on the platform in the group. Each sensor is both transmitter and receiver, which

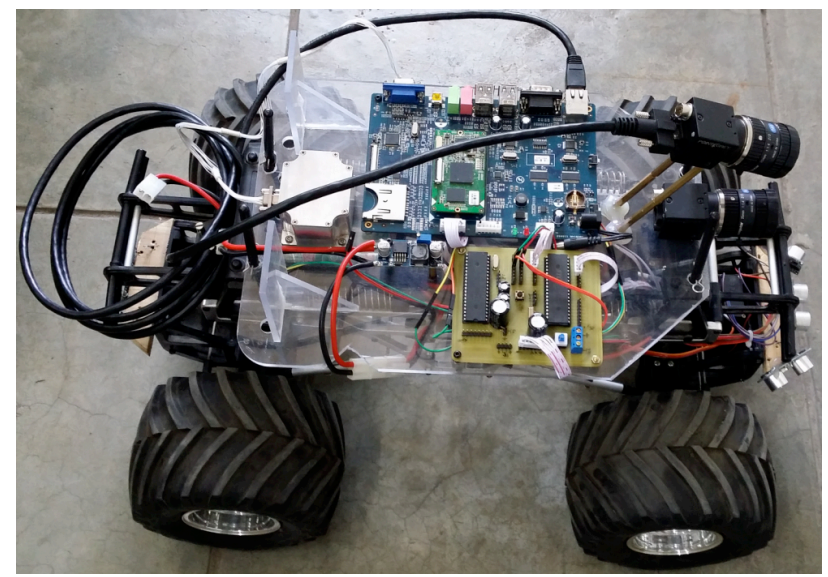

Figure 1. Test vehicle

emits ultrasonic waves and receives a return wave. In order to avoid reactive interference of the beams, the sensors transmit them in turn. The data obtained from these sensors are fused by neural network, and the actual distance is estimated. Input data of the network is the measured values from sensors, and output is the distance, from the intelligent vehicle platform front center to the measured obstacle.

\section{D-S EVIDENCE THEORY}

The information fusion based on D-S evidence theory is a decisive fusion for identification results of the neutral network to form the final decision of target attribute. D-S evidence theory fusion process is shown in Fig.2. When $\mathrm{D}-\mathrm{S}$ evidence theory is used in the decisive fusion, the data information obtained from the neutral network is the evidence in the theory, and the trust degree can be reallocated to the information. Reasoning of D-S uncertainty evidence strengthens trust degree of the obstacle region, and weakens trust degree of the empty region, which achieve accurate environment information.

\section{Simulation Results AND Discussion}

\section{A. Fusion results by BP neural network of ultrasonic ranging sensors}

Sensor readings are considered to be the shortest distance from the sensor to the obstacle in that angle. Effective range of the sensors is $1 \mathrm{~cm}-8 \mathrm{~m}$, and the sample data is randomly generated from random angle in range of $0^{\circ}$ $35^{\circ}$.The whole batch of sample data in BP neural network algorithm contains 2500 sets of group readings of the real distance between obstacles and vehicle body from sensors. 


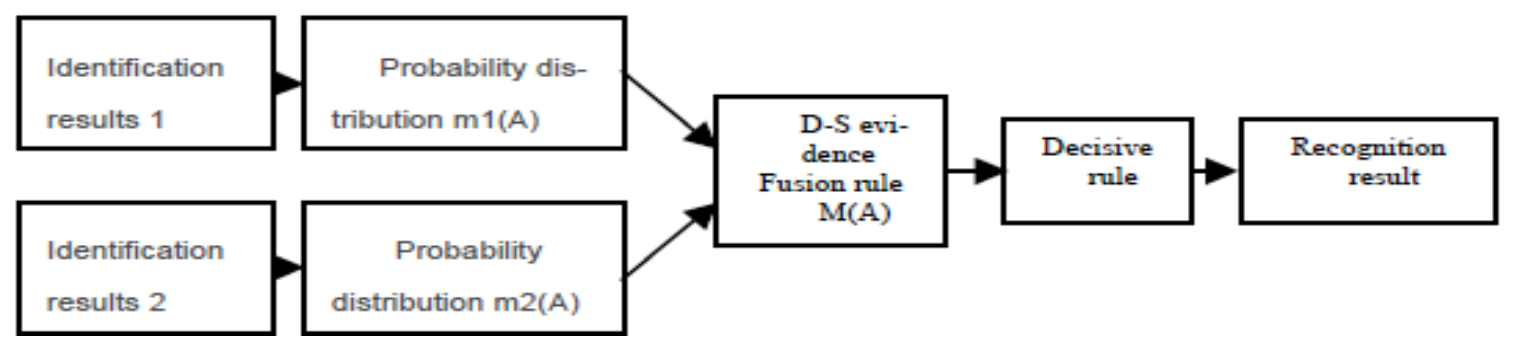

Figure 2. Fusion Process Based on D-S Evidence Theory

In simulation tests, the connection weight coefficients are randomly arranged in the range of $-1 \sim 1$. Each group of sensor data is input to the BP neural network system, and the sample data is repeatedly used to adjust connection weights until output error meets requirements .In Matlab environment, programs are written for BP neural network to train. Training times amounts to 5151 times and 5126 times, with the corresponding learning curve shown in Fig.3.Fig.3 shows that training after 1800 times meets requirements of training accuracy. The curve begins to change rapidly, and then slows down. It takes a long time to reach the minimum error state. Test data from different test are used to test the BP neural network after training, and results of the test are shown in Table I. Table I shows that performance of BP neural network has met the requirements.

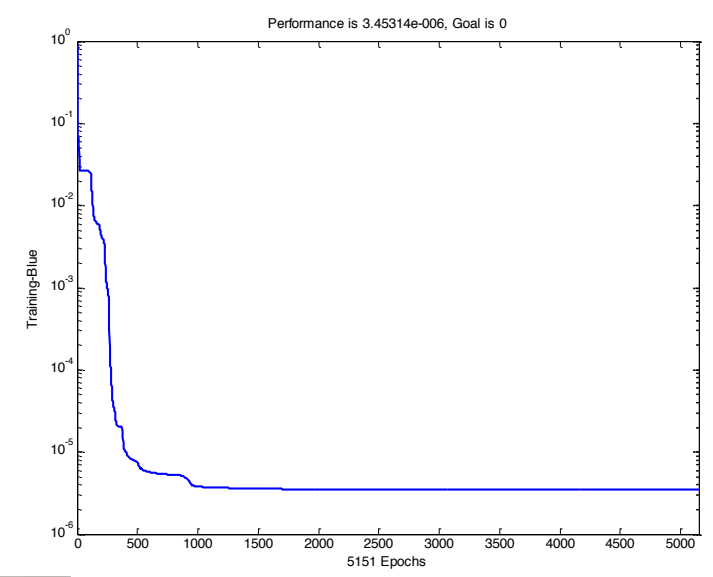

Stop Training

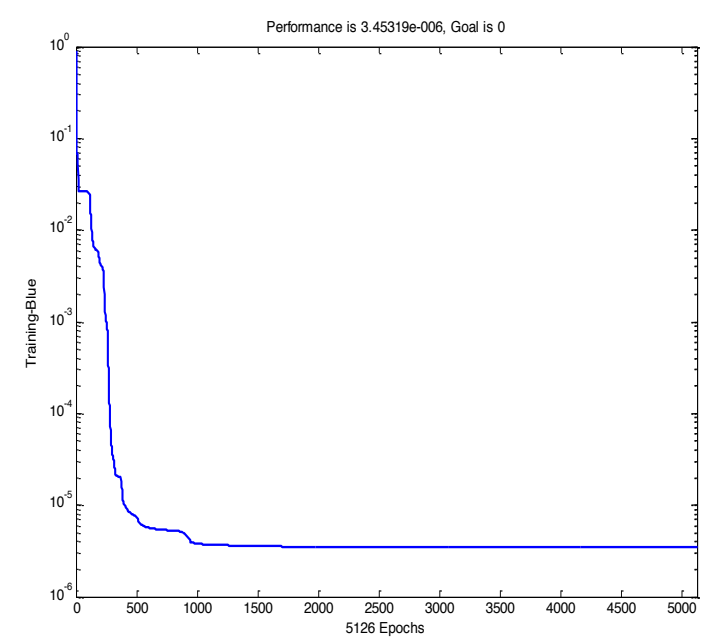

Figure 3. Training Training curve of BP network
TABLE I.

TESTING DATA

\begin{tabular}{|rccccc|}
\hline \multicolumn{3}{|c}{ Obstacle distance d0/cm } & \multicolumn{2}{c|}{ error } \\
\hline \multicolumn{4}{|c}{ Sensor reading } & $\begin{array}{c}\text { real } \\
\text { distance }\end{array}$ & $\begin{array}{c}\text { fusion } \\
\text { distance }\end{array}$ \\
$\mathrm{r} 1 / \mathrm{cm}$ \\
\hline 36.2 & 34.9 & 35.8 & 35 & 35.05 & 0.05 \\
56.8 & 55.1 & 57.2 & 55 & 55.08 & 0.08 \\
76.8 & 75.1 & 77.2 & 75 & 76.01 & 1.01 \\
101.9 & 102.8 & 103.0 & 100 & 101.03 & 1.03 \\
148.9 & 153.1 & 151.2 & 150 & 149.03 & -0.97 \\
253.4 & 247.6 & 254.3 & 250 & 252.04 & 2.04 \\
354.2 & 351.2 & 355.4 & 350 & 351.04 & 1.04 \\
454.4 & 447.6 & 453.2 & 450 & 449.07 & -0.93 \\
552.4 & 554.3 & 548.7 & 550 & 552.02 & 2.02 \\
654.1 & 655.2 & 651.2 & 650 & 651.91 & 1.91 \\
754.2 & 753.2 & 747.9 & 750 & 752.06 & 2.06 \\
\hline
\end{tabular}

B. Navigation results from D-S evidence theory decision fusion

Through the two level fusion method for obstacle recognition, and in different environment for simulation experiment, the simulation environment is shown in figure 4.

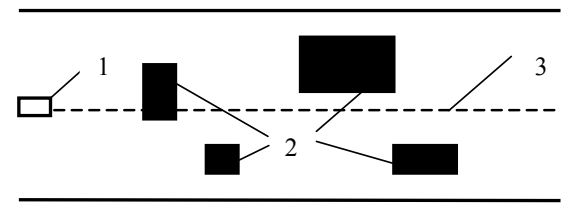

Figure 4. Navigation control simulation environment

1.Initial position of the vehicle 2. obstacles 3. mark line

Rules for intelligent vehicle tracking and obstacle avoidance:

(1)The vehicle moves along the mark line on the ground when no obstacle is observed.

(2)When obstacles are detected, the vehicle stops tracking, and starts obstacle avoidance procedures. It slows down and bypasses the obstacles according to real-time measured information on both sides of the ultrasonic sensors to determine steering range.

(3) Going around the obstacles in the road, the vehicle detects the mark line with the camera. If there is line and no obstacle in the front, the vehicle starts the tracking program, and if there are still obstacles, it continues obstacle avoidance.

In few obstacles environment of straight mark line, navigation trajectory to avoid obstacles is shown in Fig.5. The intelligent vehicle detects the obstacles with a camera, 
starts the obstacle avoidance procedures. After bypassing the obstacles, the vehicle traces the road mark line, and starts tracking program to continue driving. Navigation trajectory to avoid obstacles in multiple obstacles environment according to straight mark line is shown in Fig.6. The vehicle detects two obstacles with a camera, judges the distance between them, and tracks the way to go through the passage between the two obstacles, meanwhile it keeps safe distances on both sides from obstacles. Navigation trajectory of intelligent vehicle to avoid obstacles in multiple obstacles environment according to curve mark line is shown in Fig.7. The intelligent vehicle detects obstacles with a camera, judges the distances of the vehicle and the obstacles and between the obstacles and makes decisions to start avoidance procedure program. After the vehicle evades the multiple obstacles, it finds the mark line finally and continues tracking.

The navigation tests are carried out for methods of twolayer fusion and single-layer fusion based on fuzzy logic in two kinds of environment of few and multiple obstacles near the mark line. The intelligent vehicle moves a distance of $200 \mathrm{~m}$, and the errors of each segment tracking is shown as in Table II.

Table II shows that few obstacles around mark line lead to higher tracking accuracy of intelligent vehicle, while multiple obstacles result in increased tracking errors due to frequent turns to avoid obstacles and synchronous rear wheel driving which makes turning radius increase. In the case of few obstacles, two-layer method runs with less error than single-layer fusion, but in the case of multiple obstacles, the tracking error of two-layer method is significantly smaller than that of the latter. This is due to use of BP neural network in multiple ultrasonic sensor information fusion, which shows certain learning and adaptive ability to understand information environment multiple accurately and to eliminate or reduce uncertain information of sensors. D-S evidence theory fuses different neural network recognition results of information to avoid adverse effects to the whole system due to inaccuracy of network identification for feature information to improve the recognition effect of the system.

\section{Simulation TeST}

CarSim software with a circular bend test condition is selected for simulation conditions to be set: (1) driving speed of intelligent vehicle at $30 \mathrm{~km} / \mathrm{h}$; (2) road adhesion coefficient $\mu=0.85$ when intelligent vehicle is driven on flat road; (3) bend curvature radius of the vehicle at $152.4 \mathrm{~m}$ when it is driven on the planning path. Road track of the circular bend test is shown in Fig.8.

Tracking trajectory of the vehicle is shown in Fig.9, and tracking error of the centroid is in Fig. 10.

In Fig.9, the vehicle trajectory and the desired trajectory are basically the same, which shows that the two-level joint information fusion algorithm performs satisfactorily in tracking. In Fig. 10, lateral tracking error is controlled in $0.027 \mathrm{~m}$, of relative high control precision in the whole process of circular bend simulation test.

Steering wheel angle of the intelligent vehicle is as in Fig.11, and the steering torque is shown in Fig.12.

Fig. 11 shows that the steering angle is obtained by a front wheel angle from tracking controller multiplying the

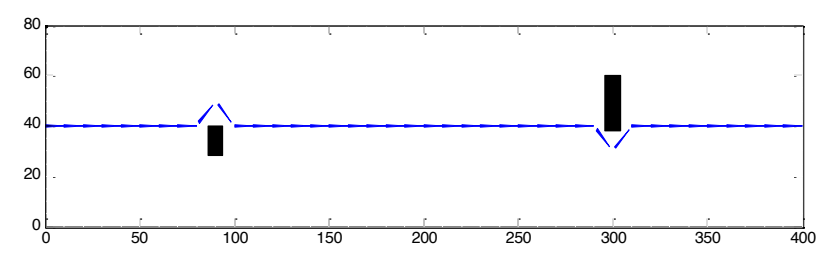

Figure 5. Trajectory in straight mark line environment of few obstacles

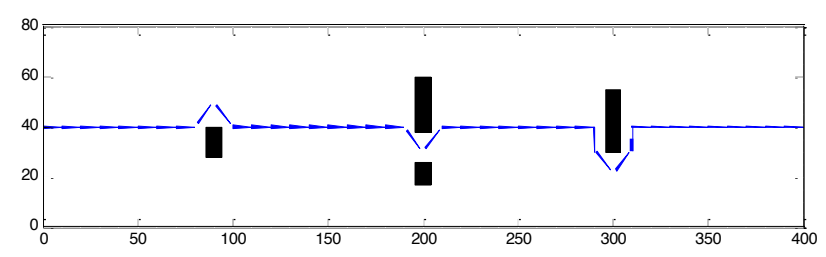

Figure 6. Trajectory in straight mark line environment of multiple obstacles

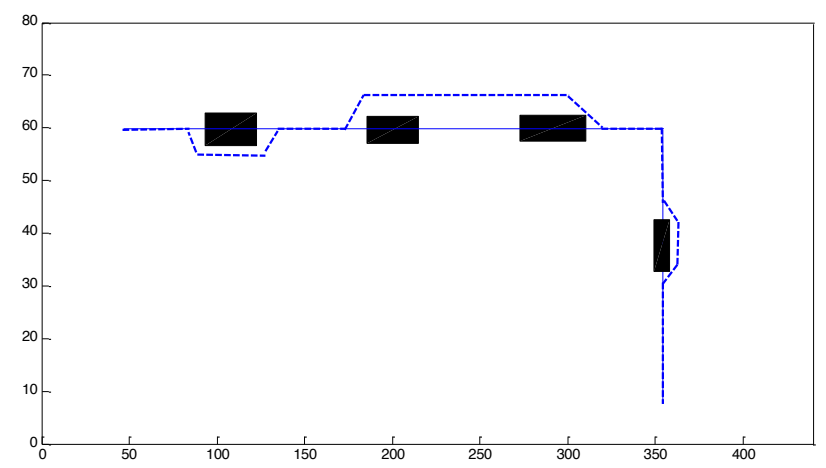

Figure 7. Trajectory in curve mark line environment of multiple obstacles

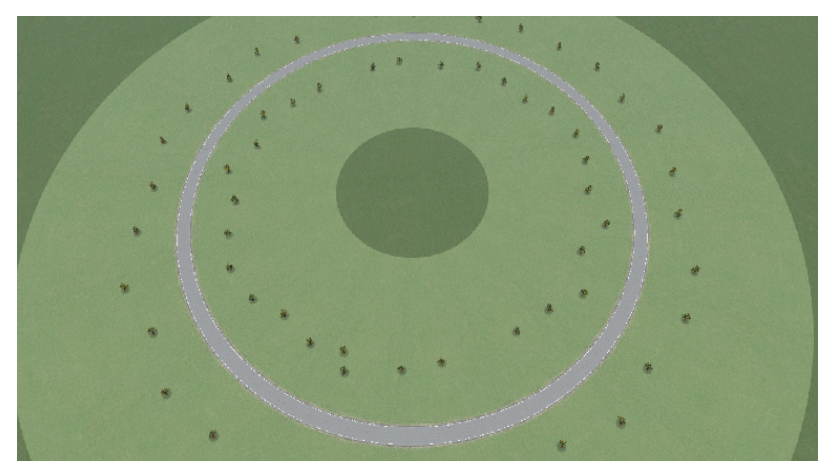

Figure 8. Road track for the circle bend simulation test

TABLE II. TRACKING ERRORS

\begin{tabular}{|c|cc|cc|}
\hline \multirow{2}{*}{ Length } & \multicolumn{2}{|c|}{ Navigation of two-layer method } & \multicolumn{2}{c|}{ Single-layer fusion method } \\
\cline { 2 - 5 } & $\begin{array}{c}\text { obstacles } \\
\text { Less }\end{array}$ & $\begin{array}{c}\text { obstacles } \\
\text { Multiple }\end{array}$ & $\begin{array}{c}\text { obstacles } \\
\text { Less }\end{array}$ & $\begin{array}{c}\text { obstacles } \\
\text { Multiple }\end{array}$ \\
\hline $0-50$ & 0.086 & 0.121 & 0.124 & 0.233 \\
$50-100$ & 0.076 & 0.132 & 0.167 & 0.287 \\
$100-150$ & 0.091 & 0.145 & 0.103 & 0.291 \\
$150-200$ & 0.101 & 0.201 & 0.187 & 0.302 \\
\hline
\end{tabular}


steering angle ratio. In Fig.12, we can see that the steering torque is very small in the process of circular bend simulation test. As the steering torque is an important parameter to characterize the steering weight, the small torque indicates a satisfactory steering operation.

Lateral acceleration of the intelligent vehicle is shown in Fig.13, and the inclination angle is in Fig.14.

In Fig.13, lateral acceleration control is in the range of $0.04648 \mathrm{~m} / \mathrm{s}^{2}$, which is enough to guarantee the comfort and stability of vehicle during the whole circular bend simulation test. And in Fig.14, side inclination angle has been limited within the range of $0.07657^{\circ}$, which shows no rollover possibilities for the vehicle in whole process of the test.

\section{CONCLUSIONS}

As for the uncertain information and the limitation of single-layer sensor information fusion in automatic navi-

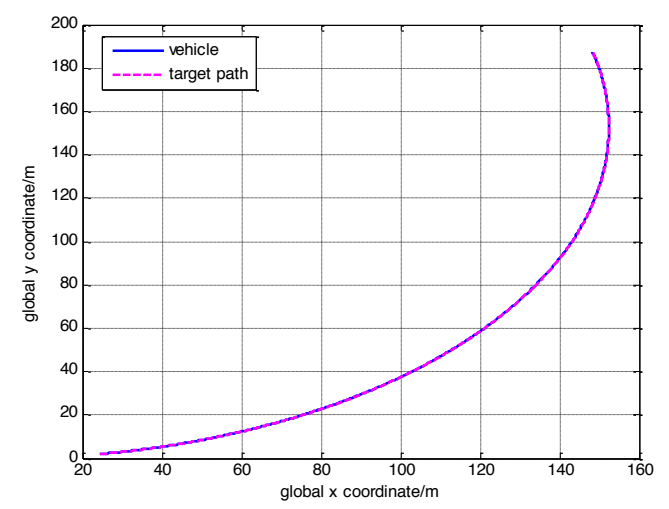

Figure 9. Trajectory tracking diagram

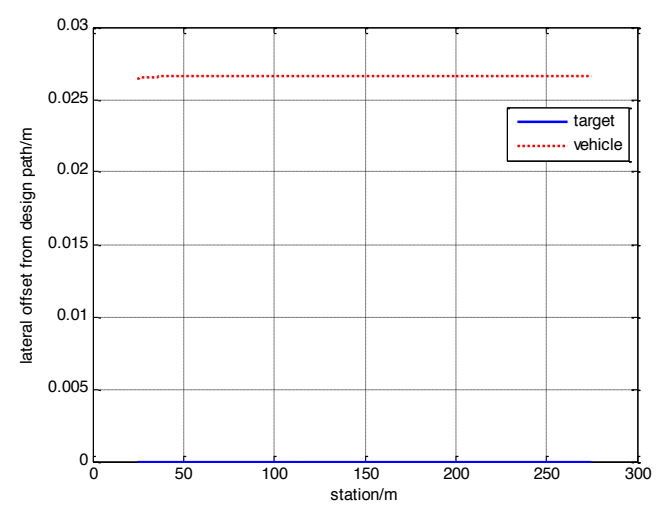

Figure 10. Lateral tracking error

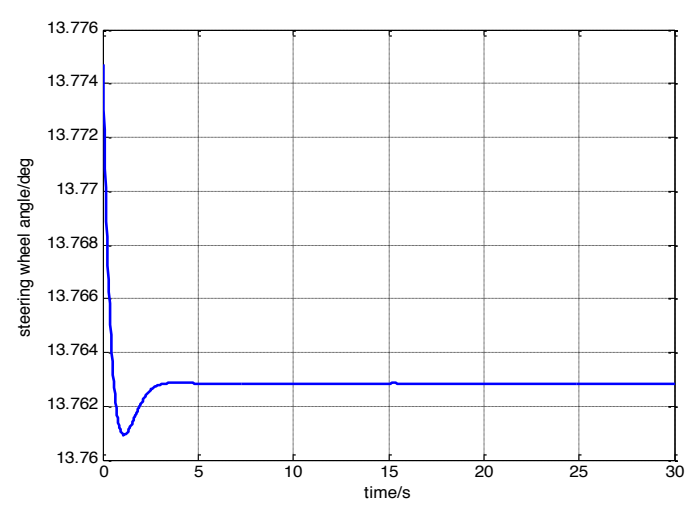

Figure 11. Steering wheel angle gation sensor detection of intelligent vehicle, the author puts forward a two-level fusion method based on combination of BP neural network and D-S evidence theory for application of intelligent vehicle tracking and obstacle avoidance control. Use of BP neural network is for information fusion of multiple ultrasonic sensors to eliminate or reduce the uncertain information from them. D-S evidence theory is to realize control of the intelligent vehicle navigation decision aiming at that decision signal is multiple suitable for control of the navigation system.

Simulated tests show that the intelligent vehicle tracks the mark line well, and avoids obstacles to complete the navigation task quickly and effectively. Compared with the traditional single-layer fusion method, the proposed information fusion scheme shows the system achieves good performance.

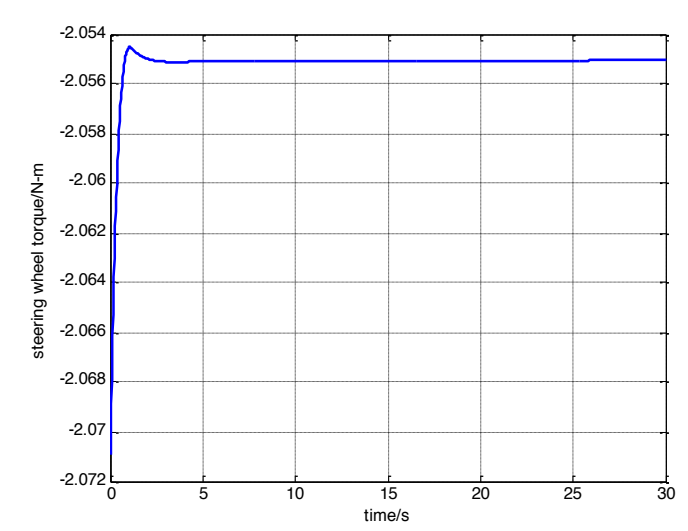

Figure 12. Steering Torque

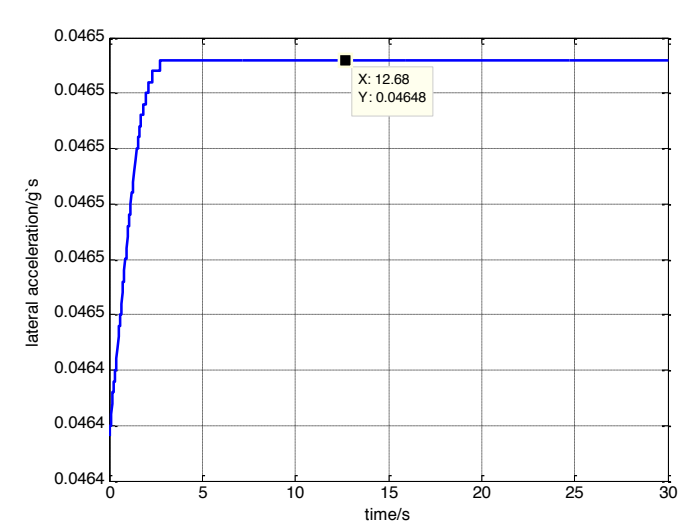

Figure 13. Lateral acceleration

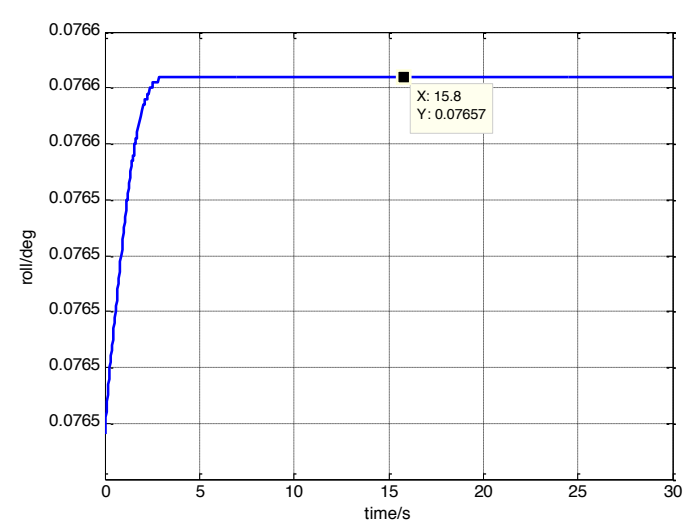

Figure 14. Side inclination angle 


\section{SHORT PAPER}

\section{APPLICATION OF TWO-LEVEL JOINT INFORMATION FUSION MODEL IN INTELLIGENT VEHICLE}

\section{REFERENCES}

[1] S. Kumar, Neural Network, Tsinghua University press, Beijing, 2006.

[2] Yuta Tsuchida and Michifumi Yoshioka, A Parallelization Method for Neural Network Learning, Electrical Engineering in Japan, vol.191, no.2, pp.17-23, 2015. http://dx.doi.org/10.1002/eej. 22694

[3] Ye Wang and Lotfollah Najjar, Factor Neural Network Theory and Its Applications, International Journal of Information Technology \& Decision Making, vol.14, no. 2, pp.239-245, 2015. http://dx.doi.org/10.1142/S0219622015500042

[4] XueMei, Qi and ShaoCong Zhang, Application of seismic multiattribute fusion method based on D-S evidence theory in prediction of CBM-enriched area, Applied Geophysics, vol.9, no.19, pp. $80-86,2012$

[5] .Peng.Li, Intuitionistic fuzzy decision-making methods based on grey incidence analysis and D-S theory of evidence, Grey Systems, Theory and Application, vol.2, no.12, pp.54-62, 2012.

[6] Fengbao,Yang and Xiaoxia,Wang,Combination Method of conflictive Evidences is D-S Evidence Theory, National Defence Industry Press, Beijing,2010.

[7] M. Young, The Technical Writer's Handbook. Mill Valley, CA: University Science, 1989.

\section{AUTHORS}

Yanting Lan, Lecturer and $\mathrm{PhD}$, major in application of data fusion and intelligent control. Address: College of Computer Science and Control Engineering, North University of China, P.C. 030051, (lytcyb@foxmail.com).

Jinying Huang, Professor and doctor supervisor, major in Analysis and control of structure dynamics and intelligent vehicle.Address: College of Mechanical and Power Engineering, North University of China, Taiyuan, P.C. 030051, (jyhuang@nuc.edu.cn).

Xiaodong Chen,College of Agriculture and Biotechnology, China Agricultural University /Key Laboratory of farming system, Ministry of agriculture, $\mathrm{PhD}$, major in application of agricultural information, intelligent agriculture application. Address: College of Agriculture and Biotechnology, China Agricultural University,Beijing P.C. 100193 (xiaodong136@foxmail.com).

This work is partially supported by Scientific and Technological Project under Grant 20130321005-04 and 20140311027-02.The authors also gratefully acknowledge the helpful comments and suggestions of the reviewers, which have improved the presentation. Submitted 19 October 2015. Published as resubmitted by the authors 20 february 2016. 